\title{
The Effect of Human Papillomavirus E6 Oncogene on the Radiosensitivity of Non-Oropharyngeal Cancer Cells
}

\author{
Angela Hong ${ }^{1,2}$, Xiaoying Zhang ${ }^{1}$, Xiao Mei Zhang ${ }^{1,2} \&$ Barbara Rose $^{3}$ \\ ${ }^{1}$ Central Clinical School, Sydney Medical School, The University of Sydney, Australia \\ ${ }^{2}$ Department of Radiation Oncology, Chris O Brien Lifehouse, Australia \\ ${ }^{3}$ Department of Infectious Diseases and Immunology, The University of Sydney, Australia \\ Correspondence: Angela Hong, Central Clinical School, Sydney Medical School, The University of Sydney, \\ Australia. E-mail: angela.hong@sydney.edu.au
}

Received: August 16, 2017

Accepted: September 3, $2017 \quad$ Online Published: September 11, 2017

doi:10.5539/cco.v6n2p7

URL: https://doi.org/10.5539/cco.v6n2p7

\begin{abstract}
Background:

We have previously shown that stable transfection of the human papillomavirus (HPV) E6*I oncogene can sensitize two HPV negative oropharyngeal cancer (OSCC) cell lines to radiation. In the current study, we extended our work on OSCC to determine whether the HPV E6 oncogene can enhance the radiosensitivity of non-OSCC cell lines.
\end{abstract}

Methods:

Three non-OSCC cell lines (melanoma, colorectal adenocarcinoma and large cell lung cancer) were stably transfection with the HPV E6 oncogene (E6 total, E6*I and E6*II) and treated with different doses of radiation. Clonogenic assays were used to measure the radiation survival.

Results:

Following transfection, there was a reduction in the survival of the melanoma cell line after 2 Gy (SF2) from 0.401 (untransfected) to 0.219 (Melanoma-E6 total). This reduction was not evident at higher doses of radiation. There was no significant change in the SF2 of melanoma-E6*I (0.303) and melanoma-E6*II (0.414). The SF2 colorectal adenocarcinoma and large cell lung cancer cell lines did not change significantly after transfection.

Conclusions:

The radiosensitizing effect of HPV E6 oncogene is cell line specific. We found no clear evidence of a radiosensitising effect of E6 in these three non-OSCC cancer cell lines.

Keyword: oropharyngeal cancer, HPV, E6, radiosensitivity

\section{Introduction}

Viral infectious diseases and cancer are both pandemics and it has been estimated that over $15 \%$ of all cancers worldwide are related to infection by virus; e.g. oropharyngeal squamous cell carcinoma (OSCC) and cervix with human papillomavirus (HPV), nasopharyngeal carcinoma with Epstein-Barr virus, and Kaposi's sarcoma with human herpes virus 8 . Radiation therapy plays an important role in the management of these viral-related malignancies and they are all relatively radiosensitive clinically. Therefore the impact of viral infection on the response of cancer to radiotherapy is critical in the field of radiation biology.

OSCC is a clinically and biologically heterogeneous disease. HPV is implicated in the majority (up to 70\%) of OSCC in the western world and this subset of OSCC demonstrates favorable prognosis due to improved response to therapy, including radiation therapy (Gillison 2004, Gillison 2006, Weinberger, Yu et al. 2006, Lassen, Eriksen et al. 2009, Hong, Dobbins et al. 2010). With increased knowledge of the role of HPV oncoproteins E6 and E7 in degrading tumour suppressor genes (p53 and pRb), there has been interest in recent years in exploring the pathways by which response to radiation are modulated. High risk HPV are DNA viruses and they encode 2 principal oncogenic open reading frames, E6 and E7, that are transforming in vitro and tumorigenic in vivo (Munger and Howley 2002). E6-containing transcripts can be spliced, yielding E6* mRNAs 
(Schneider-Gadicke and Schwarz 1986, Smotkin and Wettstein 1986). The unspliced E6 transcript gives rise to a $19 \mathrm{kDa}$ protein containing 2 putative zinc fingers, whereas E6*I and E6*II give rise to nearly identical 6kDa proteins truncated within the first zinc finger domain. Full length E6 and E6*I can cooperate with E7 and ras to transform cells in vitro, whereas E6*II cannot (Yamada, Yamashita et al. 1995). Expression of high levels of E6 in epidermal keratinocytes is sufficient to induce carcinomas, apparently acting at later stages of progression (Song, Pitot et al. 1999, Song, Liem et al. 2000). We have previously shown that stable transfection of the E6*I oncogene can sensitize two HPV negative OSCC cell lines (UM-SCC4 and WSU-HN6) to radiation (Pang, Delic et al. 2011). In the current study, we extended our work on OSCC to determine whether the HPV E6 oncogene can enhance the radiosensitivity of non-OSCC cell lines.

\section{Methods and Materials}

Cell culture, plasmids and transfections

Three non-OSCC cell lines were used: MM96L (melanoma), SW480 (colorectal adenocarcinoma, ATCC CCl-228) and H460 (large cell lung cancer, ATCC HTB-177). All three cell lines were p16 negative on immunohistochemistry indicating that they were HPV negative. The cell lines were grown in antibiotic-free Advanced RPMI 1640 (Gibco, Life technology) containing 5\% fetal calf serum and 20mM glutamine. Cells were maintained at $37^{\circ} \mathrm{C}$, in a humid environment containing $5 \% \mathrm{CO}_{2}$. Cells were removed from flasks using either $1 \mathrm{x}$ trypsin/EDTA or a scraper, then counted and dispensed for downstream experiments.

Cells were seeded at a density of $0.5 \times 10^{5}$ per well in 24-well plates. The selective agent, G418 (Gibco, Life technology) was then added to each well at doses from $0 \mu \mathrm{g}$ to $500 \mu \mathrm{g}$ per well. The minimal dose of G418 that led to $95 \%$ of death on day 3 and $100 \%$ of cell death on day 7 was defined as the cell-killing dose and used for the downstream stable transfection selection of the individual cell line. The concentrations of G418 for generating stable transfection $(900-1000) \mu \mathrm{g} / \mathrm{ml}$ were later adjusted proportionally to the different tissue culture vessels.

HPV oncogenes E6*I, E6*II and E6total (the entire open reading frame) with amino-terminal EGFP fusion expression plasmids CEFL2 (G418-selectable) were generated as previously described.(Pang, Delic et al. 2011) The plasmids were then expanded by electroporation into TOP10 E. coli competent cells (Promega). They were then grown, harvested and purified using the Plasmid Midi Kit (Qiagen). The E6-containing plasmids and the empty vector were then linearized by restriction digestion with bovine serum albumin (New England Biolabs) at $37^{\circ} \mathrm{C}$ for two hours, then heat-inactivated and examined on $1.5 \%$ TAE agarose gel for complicity. The final linearized plasmids were then used for the downstream transfection experiments.

Cells were seeded at $2 \times 10^{6}$ cells/well in 6-well plates before transfection. The cells were grown in the basic media prior to transfection until reaching $80 \%$ confluence. Transfections were then performed using the Lipofectamine-LTX Kit (Invitrogen) as described by the manufacturer, with modifications. Each $2.5 \mu \mathrm{g}$ of linearized plasmid was diluted in $500 \mu \mathrm{l}$ of fetal calf serum and antibiotic-free Opti-MEM (Invitrogen), then combined with $2.5 \mu$ of Plus Reagent at room temperature for ten minutes. Lipofectamine-LTX $(6.25 \mu l)$ was then added and allowed to form a complex at room temperature for 90 minutes. This extended incubation time was used to ensure complete complexation of all fragments in the presence of added protein contamination post-digestion. Basic medium was then added to a final volume of $2 \mathrm{ml}$. Cells were transfected at $37^{\circ} \mathrm{C}$ for 24 hours before the Lipofectamine-containing medium was replaced with fresh basic medium. The successful transfections were confirmed with numbers of cells expressing eGFP under an FL MZIII Stereomicroscope (Leica) and incubated for another 48 hours. At 72 hours post transfection, the medium was replaced with $2 \mathrm{ml}$ of selective medium containing G418 at half concentration. Cells were incubated in 6-well plates for another 48 hours and then detached, and transferred into T25 flasks with the G418 selective medium. The selective medium was changed every three days for another 12 days and the stably-transfected cells were propagated and maintained in the same G418 selective medium.

\section{Clonogenic Assays}

The seeding numbers of each cell line for different doses of radiation were first optimized using the untransfected cell lines. Cells were trypsinized, counted, and the serial dilutions of cell suspension for each cell line were made for seeding cells into T25 flasks for each radiation dose. On the day of irradiation, flasks were filled with PBS and then treated with single doses of 0 Gy, 2 Gy, 5 Gy or 10 Gy. Immediately after irradiation, the PBS was replaced with $5 \mathrm{ml}$ of fresh medium and cells were incubated for 8 to 14 days. The cells were stained with $1 \%$ methylene blue in 50\% ethanol for 30 to 60 minutes, washed with tap water, air-dried, and counted with the DCC-560 colony counter (Thermoline Scientific). Only colonies with at least 50 cells were counted. Survival curves were fitted using a linear-quadratic model. 


\section{Results}

The three cell lines that had stable expression of GFP-tagged E6 isoforms were selected by FACS. Fluorescence microscope studies of the location of E6 isoforms showed that E6total gave rise to a predominantly nuclear signal, whereas E6*I was predominantly cytoplasmic. In the majority of cells, the cytoplasmic fluorescence was punctate whereas the nuclear fluorescence was diffuse, similar to the pattern seen for HPV18 E6 in transiently transfected cells. E6*II gave a similar pattern of location to E6*I.

Figure 1 shows the radiation survival of the three cell lines with or without transfection. None of the three cell lines showed a significant change in survival after transfection with E6 total, E6*I or E6*II. Following transfection, there was a reduction in the survival of the MM96L cell line after 2 Gy (SF2) from 0.401 (untransfected) and 0.337(MM96L GFP) to 0.219 (MM96L-E6 total). This reduction was not evident at higher doses of radiation. There was no significant change in the SF2 of MM96L-E6*I (0.303) and MM96L-E6*II (0.414). The SF2 of H460 and SW480 did not change significantly after transfection.

\section{Discussion}

Radiosensitivity is mainly due to the ability of the cell to sense radiation-induced DNA damage and to control its repair. That two HPV oncoproteins, E6 and E7, can cause genomic instability seems consistent with the enhanced radiosensitivity of HPV-related OSCC. In this current study, we extended our previous work on OSCC to investigate whether the radiosensitizing effect of E6 could be transferred to another cancer cell lines. Regulation of p53 and subsequent cell cycle control are the most probable entry points for HPV induced radiosensitization. E6 binds to p53, inducing its ubiquitin-mediated break down and lowering of the p21 level. The cell cycle therefore can progress unchecked. E7 can bind to the Rb protein, directing it to proteolytic breakdown. E2F is released from the Rb protein by the inhibition of p21 and p27 and the cell cycle continues unchecked. Therefore any radiation damage would not be sufficiently repaired, and damaged cells would be more likely to die during mitosis as they progress through the cell cycle. E6 has previously been shown to sensitize mammary epithelial cells, fibroblasts and keratinocytes to chemically induced death (Xu, Meikrantz et al. 1995, Hawkins, Demers et al. 1996, Liu, McKalip et al. 2000, Liu, Zhao et al. 2007), but has been reported to have the opposite effect or no effect in other cell types exposed to ionizing radiation or DNA-damaging chemicals(DeWeese, Walsh et al. 1997, Hampson, El Hady et al. 2001, Shai, Nguyen et al. 2007). Thus, the radiosensitizing effect might be specific to a few cell types, including keratinocytes, the natural hosts for HPVs, and be dependent on the E6*I isoform.

The HPV 16 E6 gene encodes E6*I and E6*II transcripts as well as the full length E6 mRNA. The unspliced E6 transcript gives rise to a $19 \mathrm{kDa}$ protein containing two putative zinc fingers, whereas E6*I and E6*II give rise to nearly identical $6 \mathrm{kDa}$ proteins truncated within the first zinc finger domain.(Vaeteewoottacharn, Chamutpong et al. 2005) Our previous study transfecting E6 isoforms individually or in combination into HPV negative OSCC cells showed that E6*I had the greatest radiosensitizing effect and E6*II had no effect at all.(Pang, Delic et al. 2011) In the current study, we examined the effect of E6 oncoprotein on three non-OSCC cell lines to determine whether the radiosensitizing effect of E6 protein was specific to OSCC. We have shown that E6 did not confer any radiosensitizing effect on the three cancer cell lines studied, therefore the radiosensitizing effect of E6 oncoprotein is likely to be specific to OSCC cell lines.

There have been other studies to determine the effect of HPV oncoprotein on the radiosensitivity of cancer cells. A previous in vitro study using glucocorticoids to upregulate the E6/E7 expression in HPV-positive cervical cancer cells demonstrated a reduction in radiation-induced apoptosis and hence enhanced radiosensitivity.(Kamradt, Mohideen et al. 2000) Human colon carcinoma cells engineered to express E6 and E7 showed increased levels of p53 and p21 and enhanced cell cycle arrest at G1 and G2; however, there was no difference in survival after radiation.(Bol and Gregoire 2014) A study of cervix carcinoma cell lines showed no intrinsic radiosensitivity when E6 and E7 were knocked down.(Kamradt, Mohideen et al. 2000) Therefore existing data have been inconsistent, making conclusions difficult to draw.

\section{Conclusions}

In conclusion, we found no clear evidence of a radiosensitising effect of HPV E6 in the three head and neck cancer cell lines. Other known factors that determine the radiosensitivity, such as hypoxia and tumour microenvironment, are likely to play a role in radiosensitivity and will further exploration to determine the interaction between radiosensitivity and viral related factors.

\section{List of Abbreviations}

HPV: human papillomavirus 
OSCC: oropharyngeal squamous cell cancer

Gy: Gray

$\mathrm{CO}_{2:}$ Carbon dioxide

SF2: survival fraction after $2 \mathrm{~Gy}$

\section{References}

Bol, V., \& Gregoire, V. (2014). Biological Basis for increased sensitivity to radiation therapy in HPV-positive head and neck cancers. BioMed Research International. https://doi.org/10.1155/2014/696028

DeWeese, T. L., Walsh, J. C., Dillehay, L. E., Kessis, T. D., Hedrick, L., Cho, K. R., \& Nelson, W. G. (1997). Human papillomavirus E6 and E7 oncoproteins alter cell cycle progression but not radiosensitivity of carcinoma cells treated with low-dose-rate radiation. Int J Radiat Oncol Biol Phys, 37(1), 145-154. https://doi.org/10.1016/S0360-3016(96)00448-8

Gillison, M. L. (2004). Human papillomavirus-associated head and neck cancer is a distinct epidemiologic, clinical, and molecular entity. Semin Oncol, 31(6), 744-754.

https://doi.org/10.1053/j.seminoncol.2004.09.011

Gillison, M. L. (2006). Human papillomavirus and prognosis of oropharyngeal squamous cell carcinoma: implications for clinical research in head and neck cancers. J Clin Oncol, 24(36), 5623-5625. https://doi.org/10.1200/JCO.2006.07.1829

Hampson, L., El Hady, E. S., Moore, J. V., Kitchener, H., \& Hampson, I. N. (2001). The HPV16 E6 and E7 proteins and the radiation resistance of cervical carcinoma. FASEB J., 15(8), 1445-1447. https://doi.org/10.1096/fj.00-0728fje

Hawkins, D. S., Demers, G. W., \& Galloway, D. A. (1996). Inactivation of p53 enhances sensitivity to multiple chemotherapeutic agents. Cancer Res, 56(4), 892-898.

Hong, A. M., Dobbins, T. A., Lee, C. S., Jones, D., Harnett, G. B., Armstrong, B. K., ... Rose, B. R. (2010). Human papillomavirus predicts outcome in oropharyngeal cancer in patients treated primarily with surgery or radiation therapy. Br J Cancer, 103(10), 1510-1517. https://doi.org/10.1038/sj.bjc.6605944

Kamradt, M. C., Mohideen, N., Krueger, E., Walter, S., \& Vaughan, A. (2000). Inhibition of radiation-induced apoptosis lay dexamethasone in cervical carcinoma cell lines depends upon increased HPV E6/E7. The British Journal of Cancer, 82(10), 1709-1716.

Kamradt, M. C., Mohideen, N., Krueger, E., Walter, S., \& Vaughan, A. T. (2000). Inhibition of radiation-induced apoptosis by dexamethasone in cervical carcinoma cell lines depends upon increased HPV E6/E7. $\mathrm{Br} J$ Cancer, 82(10), 1709-1716.

Lassen, P., Eriksen, J. G., Hamilton-Dutoit, S., Tramm, T., Alsner, J., \& Overgaard, J. (2009). Effect of HPV-associated p16INK4A expression on response to radiotherapy and survival in squamous cell carcinoma of the head and neck. J Clin Oncol, 27(12), 1992-1998. https://doi.org/10.1200/JCO.2008.20.2853

Liu, Y., McKalip, A., \& Herman, B. (2000). Human papillomavirus type 16 E6 and HPV-16 E6/E7 sensitize human keratinocytes to apoptosis induced by chemotherapeutic agents: roles of p53 and caspase activation. J Cell Biochem, 78(2), 334-349. https://doi.org/10.1002/(SICI)1097-4644(20000801)78:2<334::AID-JCB15>3.0.CO;2-F

Liu, Z. G., Zhao, L. N., Liu, Y. W., Li, T. T., Fan, D. M., \& Chen, J. J. (2007). Activation of Cdc2 contributes to apoptosis in HPV E6 expressing human keratinocytes in response to therapeutic agents. J Mol Biol, 374(2), 334-345. https://doi.org/10.1016/j.jmb.2007.09.031

Munger, K., \& Howley, P. M. (2002). Human papillomavirus immortalization and transformation functions. Virus Res, 89(2), 213-228. https://doi.org/10.1016/S0168-1702(02)00190-9

Pang, E., Delic, N. C., Hong, A., Zhang, M., Rose, B. R., \& Lyons, J. G. (2011). Radiosensitization of oropharyngeal squamous cell carcinoma by human papillomavirus 16 oncoprotein E6 *I. Int J Radiat Oncol Biol Phys, 79(3), 860-865. https://doi.org/10.1016/j.ijrobp.2010.06.028

Schneider-Gadicke, A., \& Schwarz, E. (1986). Different human cervical carcinoma cell lines show similar transcription patterns of human papillomavirus type 18 early genes. EMBO J., 5(9), 2285-2292.

Shai, A., Nguyen, M. L., Wagstaff, J., Jiang, Y. H., \& Lambert, P. F. (2007). HPV16 E6 confers p53-dependent 
and p53-independent phenotypes in the epidermis of mice deficient for E6AP. Oncogene, 26(23), 3321-3328. https://doi.org/10.1038/sj.onc.1210130

Smotkin, D., \& Wettstein, F. O. (1986). Transcription of human papillomavirus type 16 early genes in a cervical cancer and a cancer-derived cell line and identification of the E7 protein. Proc Natl Acad Sci U S A, 83(13), 4680-4684. https://doi.org/10.1073/pnas.83.13.4680

Song, S., Liem, A., Miller, J. A., \& Lambert, P. F. (2000). Human papillomavirus types 16 E6 and E7 contribute differently to carcinogenesis. Virology, 267(2), 141-150. https://doi.org/10.1006/viro.1999.0106

Song, S., Pitot, H. C., \& Lambert, P. F. (1999). The human papillomavirus type 16 E6 gene alone is sufficient to induce carcinomas in transgenic animals. J Virol, 73(7), 5887-5893.

Vaeteewoottacharn, K., Chamutpong, S., Ponglikitmongkol, M., \& Angeletti, P. C. (2005). Differential localization of HPV16 E6 splice products with E6-associated protein. Virol J., 2, 50. https://doi.org/10.1186/1743-422X-2-50

Weinberger, P. M., Yu, Z., Haffty, B. G., Kowalski, D., Harigopal, M., ... Psyrri, A. (2006). Molecular classification identifies a subset of human papillomavirus--associated oropharyngeal cancers with favorable prognosis. J Clin Oncol, 24(5), 736-747. https://doi.org/10.1200/JCO.2004.00.3335

Xu, C., Meikrantz, W., Schlegel, R., \& Sager, R. (1995). The human papilloma virus 16 E6 gene sensitizes human mammary epithelial cells to apoptosis induced by DNA damage. Proc Natl Acad Sci U S A, 92(17), 7829-7833. https://doi.org/10.1073/pnas.92.17.7829

Yamada, T., Yamashita, T., Nishikawa, T., Fujimoto, S., \& Fujinaga, K. (1995). Biologic activity of human papillomavirus type $16 \mathrm{E} 6 / \mathrm{E} 7 \mathrm{cDNA}$ clones isolated from SiHa cervical carcinoma cell line. Virus Genes, 10(1), 15-25. https://doi.org/10.1007/BF01724293

\section{Copyrights}

Copyright for this article is retained by the author(s), with first publication rights granted to the journal.

This is an open-access article distributed under the terms and conditions of the Creative Commons Attribution license (http://creativecommons.org/licenses/by/4.0/). 